\title{
武汉地区 $\left(30.5^{\circ} \mathrm{N}, 114.4^{\circ} \mathrm{E}\right)$ 钠流星尾迹激光雷达的初步观测
}

\author{
谢秋洪 ${ }^{(12)(3)}$ ，易帆 ${ }^{(12)(3 *}$ \\ (1) 武汉大学电子信息学院, 武汉 430079; \\ (2) 地球空间环境与大地测量教育部重点实验室, 武汉 430079; \\ (3) 武汉大气遥感国家野外科学观测研究站, 武汉 430079 \\ * 联系人, E-mail: yf@whu.edu.cn
}

2009-10-21 收稿, 2009-11-09 接受

国家自然科学基金(批准号: 40674085)、长江学者计划和教育部创新研究团队(编号: PCSIRT0643)资助

\begin{abstract}
摘要 通过将 $\mathrm{Na}$ 激光雷达的积累时间设置为 $3.2 \mathrm{~s}$, 我们在武汉地区 $\left(30.5^{\circ} \mathrm{N}, 114.4^{\circ} \mathrm{E}\right)$ 对原子流 星尾迹进行了测量. 在 16 个晚上 $166 \mathrm{~h}$ 的观测时间里, 总共发现了 125 个 $\mathrm{Na}$ 流星尾迹事件. 这 些尾迹峰值密度变化范围为 4040 39170 $\mathrm{cm}^{-3}$, 平均值为 $16430 \mathrm{~cm}^{-3}$. 出现高度范围为 $77.2 \sim$ $111.6 \mathrm{~km}$, 质心高度为 $92.6 \mathrm{~km}$. 钠尾迹高度分布的上边界与同时观测到的平均钠层剖面相类

关键词

激光雷达观测 流星消融尾迹 金属层 似. 特别地, 尾迹高度柱状图最大值出现在平均钠层峰值处. 这表明流星体进入大气层后消融 产生能被地基激光雷达探测到的流星尾迹, 更多的出现在常规金属层的峰值附近. 这与早期的 $\mathrm{K}$ 和 $\mathrm{Fe}$ 尾迹的激光雷达观测结果一致. 观测发现, 偶发钠层的形成往往伴随着钠流星尾迹群, 这些尾迹出现高度与偶发钠层的高度一致.
\end{abstract}

当流星体(质量 $>10 \mu \mathrm{g}$ ) 以 $11 \sim 72 \mathrm{~km} \cdot \mathrm{s}^{-1}$ 的速度进 人大气层时, 会由于摩擦生热而蒸发并在空间上形 成流星体碎片的尾迹 ${ }^{[1]}$, 这些碎片尾迹被称为“流星 消融尾迹”. 它们能持续存在几秒钟到数十分钟, 最 终由于扩散和化学过程的消耗而融人背景大气中. 自从流星体消融被认为是中间层顶区域金属原子层 的来源以来 ${ }^{[2]}$, 研究流星消融尾迹的特征以及它们与 金属层的关系就具有特别重要的意义. 许多科学家 已经报道了通过各种不同设备观测流星注人与沉积 的结果 ${ }^{[3 \sim 15]}$.

利用共振苂光激光雷达测量流星消融尾迹, 回 波信号表现为出现在金属层密度剖面上的密度尖峰, 具有宽度窄和存在时间短的特点 ${ }^{[13]}$. 基于在 3 个不 同站点使用常规积累周期(30 s 或者更多)收集到的 $1084 \mathrm{~h} \mathrm{Na}$ 和 $\mathrm{Fe}$ 激光雷达数据, Kane 和 Gardner ${ }^{[3]}$ 首 次发表了 101 个 $\mathrm{Na}$ 尾迹和 5 个 $\mathrm{Fe}$ 尾迹的垂直分布 柱状图. 由于大部分原子流星尾迹在激光束的照射 范围内一般只存在几秒钟 $(1 \sim 5 \mathrm{~s})$, 过长的积累时间
(30 s 或者更多)将会导致正常信号中尾迹信号的严重 削弱. 因此, 许多科学家采用改进激光雷达使用更短 的积累周期(更高的时间分辨率)的方法来研究原子 流星尾迹 ${ }^{[6,7,14,15]}$. 特别地, 德国莱伯利兹大气物理研 究所(Leibniz-Institute of Atmospheric Physics (IAP) at Kühlungsborn, Germany)的科学家在自 1996 年 10 月 至 2000 年 12 月的 394 个夜晚的观测中, 利用 3 台共 振苂光激光雷达收集了 $1823 \mathrm{~h}$ 的单脉冲光子计数剖 面数据 ${ }^{[1]}$. 从这些数据中, 总共篮选出 1279 个 $\mathrm{K}, \mathrm{Na}$, $\mathrm{Ca}$ 和 $\mathrm{Fe}$ 尾迹. $\mathrm{Ca}$ 和 $\mathrm{Fe}$ 尾迹的平均探测率分别为 1.08 和 0.88 个 $/ \mathrm{h}$, 而 $\mathrm{Na}$ 和 $\mathrm{K}$ 尾迹分别为 0.75 和 0.49 个 $/ \mathrm{h}$. 研究发现难熔的金属 $(\mathrm{Ca}$ 和 $\mathrm{Fe}$ )拥有比易挥发金属 $(\mathrm{Na}$ 和 $\mathrm{K})$ 更高的探测率. 在著名的狮子座流星雨期间, 出现率并没有预料中强烈的增强, 反而, 只有少许的 增长 ${ }^{[7,15]}$. 显然, 对原子流星尾迹出现的特征进行总 结归类还需要未来更多的观测. 基于大量的尾迹数 据, von $\mathrm{Zahn}^{[1]}$ 第一次给出了 $\mathrm{K}, \mathrm{Fe}$ 和 $\mathrm{Ca}$ 尾迹的统计 高度分布图. 观测发现, $\mathrm{K}$ 和 $\mathrm{Fe}$ 尾迹最大原子密度的 
高度分布呈现一种常规 $\mathrm{K}$ 和 $\mathrm{Fe}$ 层“阴影”的形状.

中层顶区域的金属原子广泛地被认为是流星消 融的产物. 但是, 观测到的流星注人率与金属层之间 量化的关系目前还不清楚. 在 1998 年狮子座流星雨 期间，尽管观测到了大量明显的可视流星的注人，但 是在 Okinawa $\left(26^{\circ} \mathrm{N}, 127^{\circ} \mathrm{E}\right)$ 观测到的常规铁层并没有 任何明显的变化 ${ }^{[7]}$. 在巴西 Cachoeira Paulista $\left(22^{\circ} \mathrm{S}\right.$, $46^{\circ} \mathrm{W}$ ), 对 2001 年狮子座流星雨的流行雷达观测中, 探测到的电离尾迹数目只有 $11 \%$ 的增长 ${ }^{[16]}$. 然而, 在 2004 年 11 月 18 19 日的狮子座流星雨期间, 武汉 上空钠层和铁层柱密度显示出了明显的增长, 其平 均柱密度分别为邻近夜晚的 2 倍 ${ }^{[17]} . \mathrm{Na}$ 和 $\mathrm{Fe}$ 密度的 增强明显和 8 个偶发层事件相关联. 当晚, 通过同时 共体积的流星雷达观测, 得到的电离流星尾迹出现 率的时间变化和原子数密度的增强没有清晰的量化 关系. 因此我们猜测, 那些体积太小且速度太慢以至 于不能产生电离流星信号(电离尾迹和电离头信号), 而又数量庞大的微流星体可能才是中间层顶金属原 子的主要来源 ${ }^{[18]}$. 最近, Höffner 和 Friedman ${ }^{[19,20]}$ 发 现，在 $110 \mathrm{~km}$ 以上的不同金属的层上部有着显著的 相同季节特征. 此外, 层上部的金属原子密度在夏季 增强, 与偶发微流星雨的季节变化相关, 但是与大流 星雨无关. 所以, 他们认为, 消融的流星体与金属层 上部有着直接的关联.

为了研究 $\mathrm{Na}$ 原子流星尾迹的特征并揭示与中间 层顶 $\mathrm{Na}$ 层的关系, 自 2006 年 11 月以来, 我们在武 汉实现了 $3.2 \mathrm{~s}$ 短积累周期的 $\mathrm{Na}$ 激光雷达测量.

\section{1 尾迹特征}

我们使用自 2006 年 11 月 11 日至 2008 年 3 月 4 日, 总计 16 个有效夜晚, $166 \mathrm{~h}$, 约 $1.5 \times 10^{5}$ 个 $\mathrm{Na}$ 密度 剖面的 $\mathrm{Na}$ 激光雷达数据. 武汉大学 $\mathrm{Na}$ 共振苂光激光 雷达建于 2001 年, 其技术参数参见文献[21]. 考虑到 流星尾迹持续时间及雷达信号质量, 每 64 个激光脉 冲积累的光子数保存为一个光子计数剖面. 由于需 要额外的时间 $(0.8 \mathrm{~s})$ 来进行数据传输, 一次光子计数 剖面的时间分辨率为 $4 \mathrm{~s}$. Gerding 等人 ${ }^{[6]}$ 引人了雷达 绝对敏感度的概念, 其定义是: 每发射单个激光脉冲 在每个高度门 $(96 \mathrm{~cm})$ 上受一个单位数密度的原子散 射所产生的后向光子计数. 我们计算了 $90 \mathrm{~km}$ 高度附 近的雷达绝对敏感度, 其典型值约为 $1 \times 10^{-5}$ 光子计 数. 为了获得原始 $4 \mathrm{~s}$ 时间分辨率的背景光子计数剖
面，我们计算了 $5 \mathrm{~min}$ 时间间隔里单个原始剖面的 $4 \mathrm{~s}$ 平均计数剖面. 考虑到我们雷达技术剖面的特性 $(4 \mathrm{~s}$ 时间分辨率、96 $\mathrm{m}$ 高度分辨率), 我们使用如下步骤 来进行流行尾迹搜索.

第一步, 通过比较原始 $4 \mathrm{~s}$ 光子计数剖面和对应 的平均 $4 \mathrm{~s}$ 剖面, 创建一个潜在尾迹事件(potential trail events, 简称 PTEs)池 ${ }^{[14]}$. 在常规 Na 层可被忽略 的高度上, 高度门 $I$ 的信号必须大于 6.4 个光子计数 (对应光子计数率为每单位脉冲、每单位高度门 0.1 光子计数. 在常规 $\mathrm{Na}$ 层高度范围, 高度门 $I$ 上的信号 必须大于对应平均 $4 \mathrm{~s}$ 剖面光子计数的 3 倍. 满足这 个条件的事件被记录为一个 PTEs.

第二步, 通过以下标准挑选出真正的流星尾迹: (1) 在 4 个连续剖面时间窗内 $(16 \mathrm{~s})$, 出现在高度 $I$ 和 $I \pm 1$ 的事件被作为同一个流星尾迹. 在这一步骤里, 单个流星尾迹的特征参数由最强的信号中提取而来. (2) 定义统计概率“meteor level” $C_{\mathrm{k}}$, 达到 $C_{\mathrm{k}}$ 的概率 $<1 \times 10^{-6}$ (泊松分布). 在符合标准(1)所定义的 PTEs 池 里, 高度门 $I$ 上的信号大于对应的 $C_{\mathrm{k}}$ 值的事件被作为 流星尾迹.

依照这个流星尾迹搜索流程，从约 $1.5 \times 10^{5}$ 个 $\mathrm{Na}$ 密度剖面中, 总共挑选出 125 个 $\mathrm{Na}$ 流星尾迹. 图 1 提供了 2 个例子．图 1(a)显示了一个 2007 年 11月 20 21 日夜间观测到的中等强度的 $\mathrm{Na}$ 尾迹，峰值高度为
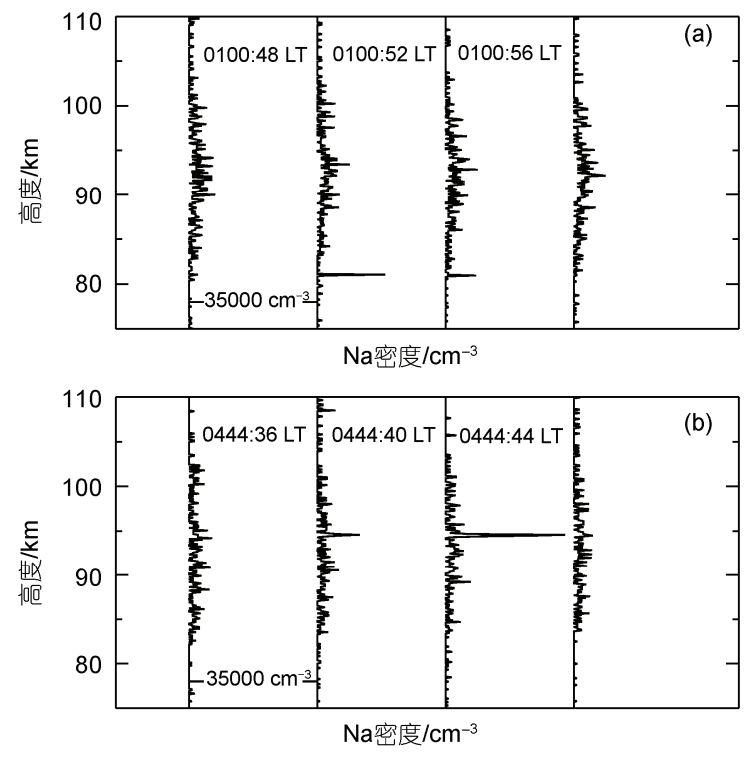

图 1 含流星消融尾迹的 $\mathrm{Na}$ 密度剖面

每个剖面高度分辨率和时间分辨率分别为 $96 \mathrm{~m}$ 和 $4 \mathrm{~s}$. 为了描述 $\mathrm{Na}$ 尾迹的演变过程, 同时画出了相邻的 $\mathrm{Na}$ 密度剖面 
$81.1 \mathrm{~km}$. 此尾迹在连续 2 个剖面持续存在, 其峰值密 度为 $1.850 \times 10^{4} \mathrm{~cm}^{-3}$, 出现时刻为 $0100: 52 \mathrm{LT}$, 柱密度 $0.14 \times 10^{9} \mathrm{~cm}^{-2}$, 约占背景 $\mathrm{Na}$ 层柱密度的 $4 \%$. 图 1 (b) 显示了一个 2007 年 11 月 21 22 日观测到的 $94.7 \mathrm{~km}$ 高 度的强流星尾迹. 此尾迹同样出现在两个连续剖面里, 峰值时刻 0444:44 LT, 峰值密度为 $3.261 \times 10^{4} \mathrm{~cm}^{-3}$, 柱密度 $0.41 \times 10^{9} \mathrm{~cm}^{-2}$, 约占背景 $\mathrm{Na}$ 层柱密度的 $13 \%$.

$\mathrm{Na}$ 流星尾迹的出现率随不同夜晚变化而变化. 在 16 个夜晚的观测中, 最大出现率为每晚 24 个尾迹, 而最小的出现率则为每晚 0 个. 这也和 von Zahn 的观 测结果一致 ${ }^{[1]}$. 表 1 给出了观测日期和对应的激光雷 达观测到的 $\mathrm{Na}$ 尾迹的数目及出现率. 为了方便比较, 同时也列出了流星尾迹和 $\mathrm{Na}$ 层柱密度的夜平均值. 从表 1 知道, 夜间流星尾迹的出现率与大流星雨时刻 表的关系并不明确. 根据计算, 我们观测到的 $\mathrm{Na}$ 流 星尾迹出现率平均每小时 0.75 个, 每晚为 7.8 个. 每 小时出现率和 von Zahn ${ }^{[1]}$ 报道的结果相同.

图 2 为 125 个 $\mathrm{Na}$ 尾迹峰值密度-高度分布、 $166 \mathrm{~h}$ $\mathrm{Na}$ 流星尾迹观测的平均 $\mathrm{Na}$ 层剖面和尾迹高度柱状 图. 为了弄清楚图 2(a)的尾迹高度分布代表的是真实 的地球物理现象还是“激光雷达仪器函数”的影响, 我们仔细检查了影响 “激光雷达仪器函数”的主要因 素—与高度相关的雷达敏感度和“meteor level” $C_{\mathrm{k}}$ 可能存在的影响. 首先, 雷达敏感度与距离的平方成 反比 ${ }^{[6]}$, 而根据我们的计算, 在距离(高度)由 $80 \mathrm{~km}$ 变化到 $100 \mathrm{~km}$ 时, 敏感度减小的因子仅仅约为 1.6 .
因此, 雷达敏感度高度变化对尾迹高度分布的影响 应当是很小的. 其次, “meteor level” $C_{\mathrm{k}}$ 在背景 $\mathrm{Na}$ 层 的峰值附近有一个较宽的极大值并且在层顶部和底 部为平坦的极小值, 因而尾迹高度分布(图 2(a))的内 沿(左边)显示出背景 $\mathrm{Na}$ 层“阴影”的形状. 我们认为, 这些强尾迹(偏离内沿的尾迹)的高度分布是由地球 物理因素决定, 而不是尾迹选择标准(“激光雷达仪器 函数”)所决定的. 因为, 在层底部和顶部范围, “meteor level” $C_{\mathrm{k}}$ 值也比较小, 那么无论尾迹强弱, 在层 顶和层底应该都能被观测到. 但是, 我们的测量结果 (图 2(a))与 von Zahn 所报道的一样，相对于背景金属 层峰值高度附近, 在层底部和顶部(约 80 和 $105 \mathrm{~km}$ 附近), 所发现的流星尾迹数目较少, 并且密度也较 小 ${ }^{[1]}$. 根据以上分析, 我们认为激光雷达观测的流星 尾迹高度分布整体(除了尾迹高度分布的内沿)反映 的是地球物理现象, 而不是 “激光雷达仪器函数”. 如 图 2(a)所示, $\mathrm{Na}$ 流星尾迹出现高度范围为 77.2 111.6 $\mathrm{km}$, 质心高度为 $92.6 \mathrm{~km}$. 峰值密度变化范围为 4040 $\mathrm{cm}^{-3} \sim 39170 \mathrm{~cm}^{-3}$, 平均峰值密度为 $16430 \mathrm{~cm}^{-3}$. 考虑 到原子尾迹在雷达视场里的持续时间通常小于一个 积累周期, 因此推测所计算得到的峰值密度被低估 了. 由图可知, 强的 $\mathrm{Na}$ 尾迹倾向于出现在背景 $\mathrm{Na}$ 层 峰值附近, 而弱的尾迹则出现在层底和层顶. 在背景 层上下边缘 (75 80 和 105 120 km), $\mathrm{Na}$ 尾迹明显较为 缺乏. 同样的特征也可以在 von Zahn 报道的 $\mathrm{K}$ 和 $\mathrm{Fe}$ 尾迹高度分布上看到 ${ }^{[1]}$. 激光雷达观测到的原子尾迹

表 1 武汉 16 个夜晚观测的 $\mathrm{Na}$ 尾迹数目、出现率、流星尾迹与 $\mathrm{Na}$ 层的平均柱密度

\begin{tabular}{|c|c|c|c|c|}
\hline 观测时间 & 尾迹数目 & 出现率(trail/h) & 尾迹平均柱密度 & $\mathrm{Na}$ 层平均柱密度 $\left(10^{9} \mathrm{~cm}^{-3}\right)$ \\
\hline 2006 年 11 月 11 12 日 & 4 & 0.44 & 0.23 & 1.99 \\
\hline 2007 年 4 月 19 20 日 & 10 & 1.05 & 0.07 & 1.56 \\
\hline 2007 年 11 月 $2 \sim 3$ 日 & 10 & 0.91 & 0.13 & 3.35 \\
\hline 2007 年 11 月 20 21 日 & 24 & 2.09 & 0.11 & 3.51 \\
\hline 2007 年 11 月 $21 \sim 22$ 日 & 12 & 1.04 & 0.14 & 2.45 \\
\hline 2007 年 11 月 22 23 日 & 1 & 0.13 & 0.19 & 2.10 \\
\hline 2007 年 11 月 $28 \sim 29$ 日 & 2 & 0.19 & 0.13 & 2.41 \\
\hline 2007 年 12 月 13 14 日 & 4 & 0.40 & 0.29 & 4.03 \\
\hline 2007 年 12 月 29 30 日 & 4 & 0.36 & 0.22 & 2.60 \\
\hline 2007 年 12 月 31 日 2008 年 1 月 1 日 & 0 & 0.00 & 0.00 & 3.15 \\
\hline 2008 年 1 月 $1 \sim 2$ 日 & 12 & 1.04 & 0.21 & 5.02 \\
\hline 2008 年 1 月 $2 \sim 3$ 日 & 9 & 0.78 & 0.17 & 3.60 \\
\hline 2008 年 2 月 $16 \sim 17$ 日 & 0 & 0.00 & 0.00 & 3.32 \\
\hline 2008 年 2 月 29 日 3 月 1 日 & 23 & 2.30 & 0.25 & 4.26 \\
\hline 2008 年 3 月 $2 \sim 3$ 日 & 8 & 1.23 & 0.26 & 4.29 \\
\hline 2008 年 3 月 $3 \sim 4$ 日 & 2 & 0.18 & 0.26 & 2.86 \\
\hline
\end{tabular}



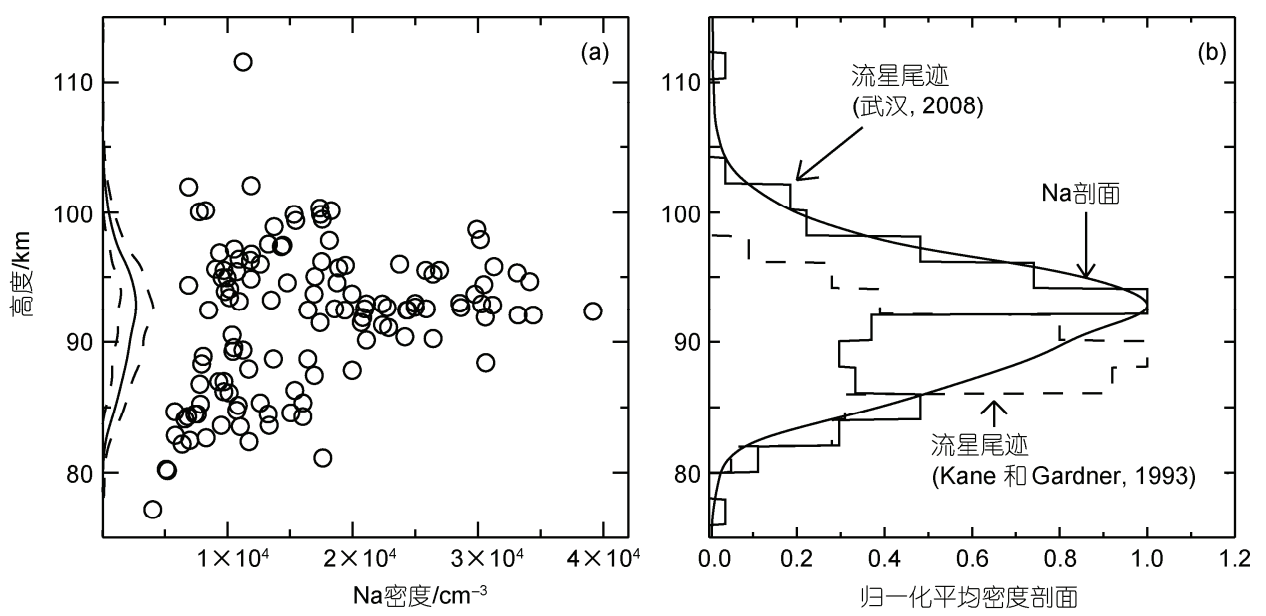

图 2 武汉激光雷达观测的 $\mathrm{Na}$ 流量尾迹统计特征

(a) 125 个 $\mathrm{Na}$ 流星尾迹(圆圈)峰值密度的高度分布. 为了比较, 提供了 $166 \mathrm{~h}$ 的平均背景 $\mathrm{Na}$ 层密度剖面(实线为常规 $\mathrm{Na}$ 层剖面, 两条虚线分别 为加上和减去均方误差的结果). (b) 武汉 125 个流星尾迹的高度柱状图、归一化平均 $\mathrm{Na}$ 层密度剖面与 Kane 和 Gardner 的结果对比

通常被认为是反映了流星体消融，随后的化学消耗 和扩散的综合结果. 根据 Kane 和 Gardner ${ }^{[3]}$ 的估计, 原子流星尾迹从消融到被激光雷达探测到之前，通 常只有几秒钟，最多几分钟的时间. 在如此短的时间 内，扩散对原子流星尾迹的影响非常弱，因为在中间 层顶区域扩散的时间尺度大于 $2 \times 10^{3} \mathrm{~s}^{[22]}$, 而化学耗 散的比率是跟高度是强相关的. 根据目前的 $\mathrm{Na}$ 气相 化学, $\mathrm{Na}$ 原子的生存时间(化学特征时间)在 $<80 \mathrm{~km}$ 的高度上非常小 $(80 \mathrm{~km}$ 处约为 $9 \mathrm{~s})$, 而在 $>100 \mathrm{~km}$ 的 高度非常大 $\left(100 \mathrm{~km} \text { 出约为 } \sim 5 \times 10^{3} \mathrm{~s}\right)^{[22]}$. 因此, 至少 在背景层上部, 流星尾迹分布实际上是由流星体消 融直接决定的 (进人流星体的速度和质量分布 $)^{[3]}$. 激 光雷达观测到的尾迹高度分布(图 2(a)和(b))显示, 流 星体进人大气之后, 倾向于在背景层峰值附近产生 更多更强的原子流星尾迹, 而不是在其他高度. 另外, 尾迹的高度柱状图(图 2(b))显示出一个双峰结构, 高 度分别为 93 和 $85 \mathrm{~km}$. 这个结果和 Kane 和 Gardner ${ }^{[3]}$ 的结果又有所不同. 考虑到其 $30 \mathrm{~s}$ 的时间分辨率相 对大部分流星尾迹过长, 我们推测, Kane 和 Gardner ${ }^{[3]}$ 报道的流星尾迹高度分布更接近于常规金属层，而 不是代表流星消融的真实情况. 图 2(b)显示的双峰结 构可能代表了在我们观测时期内, 流星体消融的两 个主要区域. 因此, 要弄清楚两个观测结果的不同, 还需要不同站点更多时间的观测.

表 2 给出了 $\mathrm{Na}$ 流星尾迹与 $\mathrm{Na}$ 层特征参数的对 比. 注意到, $\mathrm{Na}$ 尾迹分布比平均 $\mathrm{Na}$ 层剖面的质心高
度高 $0.6 \mathrm{~km}$, 而 RMS 宽度比平均 $\mathrm{Na}$ 层窄 $0.2 \mathrm{~km}$. Na 尾迹分布的质心高度和 LAP Na 激光雷达得到的 92.4 $\mathrm{km}$ 相近 ${ }^{[1]}$. 另外, 值得关注的是, $\mathrm{Na}$ 尾迹的平均峰值 密度约为背景 $\mathrm{Na}$ 层的 6 倍, 然而, 平均柱密度只占 背景 $\mathrm{Na}$ 层的 $5 \%$ 左右.

\section{$2 \mathrm{Na}$ 流星尾迹与 $\mathrm{Na}$ 层的关系}

激光雷达观测的流星尾迹代表了流星体消融在 上层大气中的中性原子的直接沉积. 但是, 根据高时 间分辨率 $\mathrm{Na}$ 激光雷达的 $166 \mathrm{~h}$ 测量, 流星尾迹出现 率与常规 $\mathrm{Na}$ 层柱密度之间量化的关系还是模糊不明 的. 一方面, 通过表 1 里相邻夜晚观测结果之间的比 较, 在较高的尾迹出现率的夜晚, 对应的夜平均 $\mathrm{Na}$ 层柱密度也相对较大(例如 2007 年 11 月 20 21 日). 另一方面，在 2007 年 12 月 31 日至 2008 年 1 月 1 日 夜晚, 尽管相比邻近夜晚(2007 年 12 月 29 30 日)要 高, 但是在 $11.5 \mathrm{~h}$ 的激光雷达观测中, 却没有发现流 星尾迹. 相比 $\mathrm{Na}$ 层的持续存在, $\mathrm{Na}$ 流星尾迹表现为 一种偶发的(间歇的)事件. 因此, $\mathrm{Na}$ 流星尾迹与背景 $\mathrm{Na}$ 层之间并没有确定的量化关系是并不奇怪的.

表 $2 \mathrm{Na}$ 流星尾迹特征参数与平均 $\mathrm{Na}$ 层的对比

\begin{tabular}{ccc}
\hline 参数 & $\mathrm{Na}$ 流星尾迹 & 平均 $\mathrm{Na}$ 层 \\
\hline 质心高度 $/ \mathrm{km}$ & 92.6 & 92.0 \\
$\mathrm{RMS}$ 宽度 $/ \mathrm{km}$ & 4.7 & 4.9 \\
平均峰值密度 $/ 10^{3}$ & 16.4 & 2.7 \\
平均柱密度 $/ 10^{9} \mathrm{~cm}^{-2}$ & 0.17 & 3.13 \\
\hline
\end{tabular}


根据高时间分辨率的 $\mathrm{Na}$ 激光雷达测量, 我们发 现，几个强 $\mathrm{Na}$ 流星尾迹和偶发 $\mathrm{Na}$ 层有明显的关联. 偶发层典型特征为在很窄的高度范围内密度增强(密 度等于或者大于背景 $\mathrm{Na}$ 层的两倍) ${ }^{[23]}$. 它们与背景 $\mathrm{Na}$ 层没有清晰的关系. 一个典型的例子是在 2008 年 3 月 3 日夜晚, 一个中等强度的偶发 $\mathrm{Na}$ 层出现在约 $95 \mathrm{~km}$ 高度附近. 起始时刻为 $0130 \mathrm{LT}$, 峰值密度达 到最大 $9390 \mathrm{~cm}^{-3}$ 的时间是 $0440 \mathrm{LT}$. 随后, 偶发层被 消耗并在 0550 LT 消失. 在这个偶发层的形成过程中, 总共有 6 个 $\mathrm{Na}$ 流星尾迹(圆圈表示)出现在偶发层峰 值高度处. 为了详细表示两者的关系, 图 3 显示了偶 发 $\mathrm{Na}$ 层期间出现的流星尾迹和对应 $5 \mathrm{~min}$ 积累的 $\mathrm{Na}$ 密度剖面. 特别的是, 最强的尾迹(峰值密度为 33940 $\mathrm{cm}^{-3}$ )出现在偶发层峰值密度接近最大值的时刻. 因 此, 这 6 个 $\mathrm{Na}$ 流星尾迹被认为和偶发 $\mathrm{Na}$ 层相关.

\section{3 总结}

自 2006 年 11 月以来, 武汉大学 $\mathrm{Na}$ 激光雷达采 用 $3.2 \mathrm{~s}$ 积累周期, 对 $\mathrm{Na}$ 原子流星尾迹进行了研究, 并对其与中间层顶 $\mathrm{Na}$ 金属层的关系进行了探索. 从
16 个夜晚 $166 \mathrm{~h}$ 的 $\mathrm{Na}$ 观测数据中, 提取出了 125 个 $\mathrm{Na}$ 流星尾迹事件. 其峰值密度范围为 4040 39170 $\mathrm{cm}^{-3}$, 平均值为 $16430 \mathrm{~cm}^{-3}$. 尾迹出现高度范围 77.2 $111.6 \mathrm{~km}$, 质心高度为 $92.6 \mathrm{~km}$. Na 尾迹分布质心高 度比平均 $\mathrm{Na}$ 层高 $0.6 \mathrm{~km}$, 而 RMS 宽度窄 $0.2 \mathrm{~km}$. Na 尾迹高度分布特征(图 2(a)中内沿和外沿)与 von $\mathrm{Zahn}^{[1]}$ 报道的 $\mathrm{K}$ 和 $\mathrm{Fe}$ 相似. 不同金属原子具有相同 特征的现象表明，地基激光雷达所探测到的，进人大 气层的流星体更倾向于在金属层峰值高度附近消融 并产生更多的原子流星尾迹. 值得注意的是, 这些 $\mathrm{Na}$ 尾迹平均峰值密度为背景 $\mathrm{Na}$ 层的 6 倍, 而平均柱密 度仅为背景 $\mathrm{Na}$ 层的 $5 \%$. 在我们观测的 $30^{\circ} \mathrm{N}$ 站点, $\mathrm{Na}$ 流星尾迹存在于从 1900 0600 LT 激光雷达观测时内, 平均出现率为 0.75 trails $/ \mathrm{h}$, 但是, 尾迹的出现随着不 同观测夜晚不同. 尽管 $\mathrm{Na}$ 流星尾迹(出现时间和强度) 与背景 $\mathrm{Na}$ 层之间量化的关系还不清晰，但是尾迹与 偶发 $\mathrm{Na}$ 层之间存在一个直接的关联. 我们的观测结 果发现, 强的偶发 $\mathrm{Na}$ 层的形成偶尔也伴随着一个 $\mathrm{Na}$ 流星尾迹群，这些流星尾迹出现在偶发 $\mathrm{Na}$ 层峰值附 近的高度.

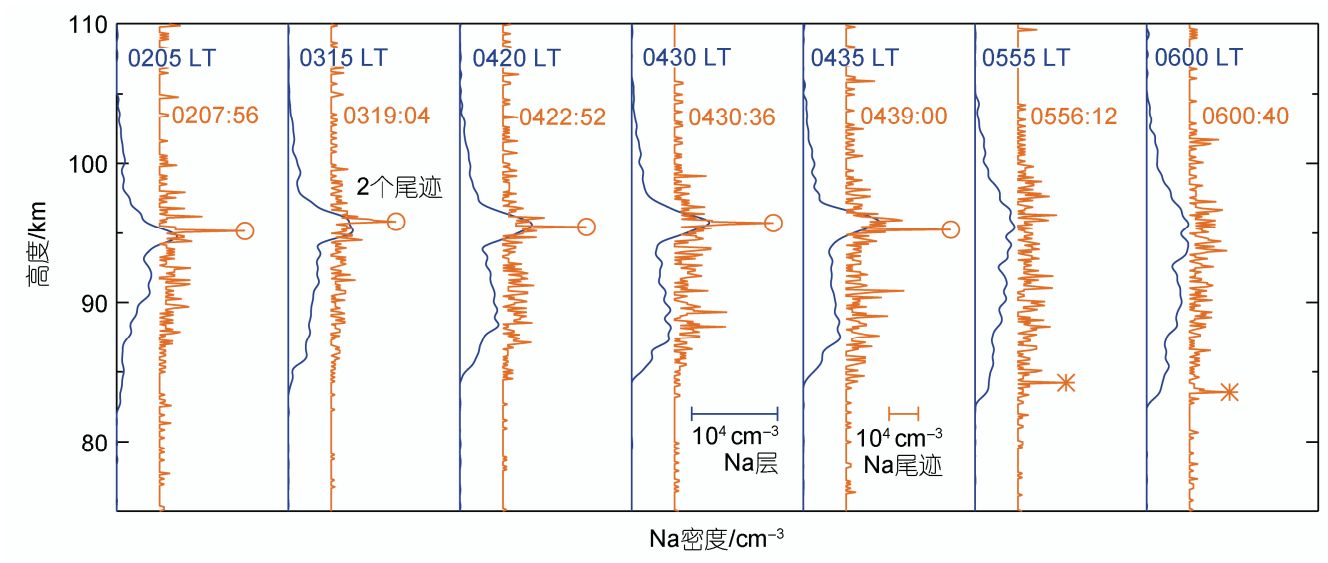

图 32008 年 3 月 3 日偶发期间 $5 \mathrm{~min}$ 积累的 $\mathrm{Na}$ 密度剖面与对应时间内流星尾迹

$3.2 \mathrm{~s}$ 积累周期. $5 \mathrm{~min}$ 密度剖面使用了 $0.5 \mathrm{~km}$ FWHM 的 Hamming 窗平滑. 包含有 2 个尾迹的, 只画出最强的尾迹所在剖面. 总共有 6 个 $\mathrm{Na}$ 流 星尾迹(圆圈)出现在偶发 $\mathrm{Na}$ 层峰值附近, 2 个尾迹出现在没有偶发 $\mathrm{Na}$ 层的时刻(星号)

1 von Zahn U. Lidar observations of meteor trails: Evidence for fragmentation of meteoroids and their subsequent differential ablation. In: Proceedings of the Meteoroids 2001 Conference. Kiruna, Sweden: Swedish Institute of Space Physics, 2001. 303-304 
2 Clemesha B R, Kirchhoff V, Simonich D, et al. Evidence of an extra-terrestrial source for the mesospheric sodium layer. Geophys Res Lett, 1978, 5: 873-876

3 Kane T J, Gardner C S. Lidar observations of the meteoric deposition of mesospheric metals. Science, 1993, 259: 1297-1300

4 Zhou Q, Tepley C A, Sulzer M P. Meteor observations by the Arecibo $430 \mathrm{MHz}$ incoherent scatter radar-I. Results from time-integrated observations. J Atmos Terr Phys, 1995, 57: 421—431

5 Zhou Q, Kelley M C. Meteor observations by the Arecibo $430 \mathrm{MHz}$ incoherent scatter radar. II. Results from the time-resolved observations. J Atmos Terr Phys, 1997, 59: 739-752

6 Gerding M, Alpers M, Höffner J, et al. Simultaneous K and Ca lidar observations during a meteor shower on March 6-7, 1997, at Kühlungsborn, Germany. J Geophys Res, 1999, 104: 24689_24698

7 Chu X, Pan W, Papen G, et al. Characteristics of Fe ablation trails observed during the 1998 leonid meteor shower. Geophys Res Lett, 2000, 27: 1807-1810

8 Chu X, Liu A, Papen G, et al. Lidar observations of elevated temperatures in bright chemiluminescent meteor trails during the 1998 leonid shower. Geophys Res Lett, 2000, 27: 1815-1818

9 Kelley M, Gardner C S, Drummond J, et al. First observations of long-lived meteor trains with resonance lidar and other optical instruments. Geophys Res Lett, 2000, 27: 1811-1814

10 Mathews J, Janches D, Meisel D, et al. The micrometeoroid mass flux into the upper atmosphere: arecibo results and a comparison with prior estimates. Geophys Res Lett, 2001, 28: 1929-1932

11 刘进波, 易帆. 2001 年狮子座流星雨期间钠苂光激光雷达的观测. 科学通报, 2003, 48: 2380-2383

12 熊建刚, 万卫星, 宁百齐, 等. 武汉上空中层顶附近大气环流的流星雷达观测. 科学通报, 2003, 48: 1102一-1106

13 Beatty T, Bills R, Kwon K, et al. CEDAR lidar observations of sporadic Na layers at urbana, illinois. Geophys Res Lett, 1988, 15: 11371140

14 Höffner J, von Zahn U, McNeil W, et al. The 1996 Leonid shower as studied with a potassium lidar: Observations and inferred meteoroid sizes. J Geophys Res, 1999, 104: 2633-2643

15 von Zahn U, Gerding M, Höffner J, et al. Iron, calcium, and potassium atom densities in the trails of Leonids and other meteors: Strong evidence for differential ablation. Meteor Planet Sci, 1999, 34: 1017-1027

16 Clemesha B R, Batista P P. Leonids meteor rates, 1999-2001. Adv Space Res, 2003, 33: 1491—1495

17 Yi F, Zhang S, Yu C, et al. Simultaneous observations of sporadic Fe and Na layers by two closely colocated resonance fluorescence lidars at Wuhan $\left(30.5^{\circ} \mathrm{N}, 114.4^{\circ} \mathrm{E}\right)$, China. J Geophys Res, 2007, 112: D04303, doi: 10.1029/ 2006JD007413

18 Yi F, Zhang S, Yue X, et al. Some ubiquitous features of the mesospheric Fe and Na layer borders from simultaneous and commonvolume Fe and Na lidar observations. J Geophys Res, 2008, 113: A04S91, doi:10.1029/2007JA012632

19 Höffner J, Friedman J S. The mesospheric metal layer topside: A possible connection to meteoroids. Atmos Chem Phys, 2004, 4: 801808

20 Höffner J, Friedman J S. The mesospheric metal layer topside: Examples of simultaneous metal observations. J Atmos Sol-Terr Phys, 2005, 67: 1226-1237

21 Yi F, Zhang S, Zeng H, et al. Lidar observations of sporadic Na layers over Wuhan $\left(30.5^{\circ} \mathrm{N}, 114.4^{\circ} \mathrm{E}\right)$. Geophys Res Lett, 2002, 29: 1345 , doi:10.1029/2001GL014353

$22 \mathrm{Xu} \mathrm{J,} \mathrm{Smith} \mathrm{A} \mathrm{K.} \mathrm{Perturbations} \mathrm{of} \mathrm{the} \mathrm{sodium} \mathrm{layer:} \mathrm{Controlled} \mathrm{by} \mathrm{chemistry} \mathrm{or} \mathrm{dynamics?} \mathrm{Geophys} \mathrm{Res} \mathrm{Lett,} \mathrm{2003,} \mathrm{30:} \mathrm{2056,} \mathrm{doi:}$ $10.1029 / 2003 \mathrm{GL} 018040$

23 Clemesha B R. Sporadic neutral metal layers in the mesosphere and lower thermosphere. J Atmos Terr Phys, 1995, 57: 725-736 